\title{
SHG/TPEF-based image technology improves liver fibrosis assessment of minimally sized needle biopsies
}

\author{
Bingqiong Wang ${ }^{1} \cdot$ Yameng Sun ${ }^{1}$. Jialing Zhou ${ }^{1} \cdot$ Xiaoning $\mathrm{Wu}^{1} \cdot$ Shuyan Chen ${ }^{1} \cdot$ Yiwen Shi $^{1} \cdot$ Shanshan $\mathrm{Wu}^{1}$. \\ Hui Liu ${ }^{2} \cdot$ Yayun Ren $^{3} \cdot$ Xiaojuan Ou' ${ }^{1}$ Jidong Jia ${ }^{1} \cdot$ Hong You' ${ }^{1}$
}

Received: 24 November 2018 / Accepted: 18 May 2019 / Published online: 11 June 2019

(c) The Author(s) 2019

\begin{abstract}
Background and aims Sampling size variability of liver biopsy remains a major limitation in the assessment of liver fibrosis. We aimed to evaluate the diagnostic value of a fully quantitative method (second harmonic generation/two-photon excitation fluorescence, SHG/TPEF based) in "short" liver biopsy samples.

Methods Liver biopsy samples from chronic hepatitis B (CHB) patients were constructed into "virtual" biopsies with different lengths. The original and "virtual" samples were measured by SHG/TPEF-based technology to obtain qFibrosis score, respectively. Here, $\Delta$ qFibrosis was defined as difference of qFibrosis between original biopsy and "virtual" biopsy. Equivalence test was used to compare $\Delta$ qFibrosis with the clinically acceptable error (deviation of 0.50 ) in each group.

Results In real-world practice, qFibrosis score increased significantly with fibrosis progression in $\geq 1.5-\mathrm{cm}-, 1.0-1.5-\mathrm{cm}-$, and $0.5-1.0$-cm-long specimens $(p<0.05)$, compared with $\leq 0.5$-cm-long specimens $(p>0.05)$. In virtual biopsy samples with specified length, the equivalence was confirmed in $0.5-1.0-\mathrm{cm}-$ and $1.0-1.5-\mathrm{cm}-$ long specimens $(0.27 \mathrm{vs} .0 .22, p<0.001)$, whereas not in $\leq 0.5$-cm-long specimens $(0.53, p>0.05)$. The number of cross-linked collagen fibers, the total and aggregated collagen proportionate area, and the collagen strings in number, length, width and perimeter showed excellent consistency with original biopsy samples in $0.5-1.0-\mathrm{cm}$ - and $1.0-1.5$-cm-long specimens (ICC $>0.90$ ).

Conclusions The use of SHG/TPEF-based image technology may give useful suggestive information in evaluation of CHBrelated liver fibrosis for the short sample (biopsy length $>0.5 \mathrm{~cm}$ ).
\end{abstract}

Keywords Liver fibrosis $\cdot$ Liver biopsy $\cdot$ Sampling error · Quantification

Electronic supplementary material The online version of this article (https://doi.org/10.1007/s12072-019-09955-2) contains supplementary material, which is available to authorized users.

Hong You

youhong30@sina.com

1 Liver Research Center, Beijing Key Laboratory of Translational Medicine in Liver Cirrhosis, National Clinical Research Center for Digestive Disease, Beijing Friendship Hospital, Capital Medical University, 95 Yong-an Road, Xi-Cheng District, Beijing 100050, China

2 Pathology Department, Beijing Youan Hospital, Capital Medical University, Beijing, China

3 Hangzhou Choutu Technology, Hangzhou, China

\section{Introduction}

Liver biopsy has been widely approved as the gold standard in evaluating liver fibrosis for patients with chronic liver disease. However, sampling variability continues to be one of the limitations of liver biopsy in the assessment of liver fibrosis [1-4].

Many studies tried different methods to overcome this limitation. A biopsy specimen with sufficient size has been recommended to minimize sampling error and to improve the diagnostic accuracy of liver biopsies. Whereas, the optimal length remains controversial [5, 6], in an early study, a biopsy length of $1.5 \mathrm{~cm}$ was considered adequate [5]. Thereafter, an optimal biopsy sample length of $2.0 \mathrm{~cm}$ and $2.5 \mathrm{~cm}$ was recommended [4] [7].

However, sufficient sample size cannot be guaranteed for each liver biopsy in clinical practice. Biopsy length data indicate that biopsies smaller than the current recommendations 
are obtained in over half of the patients [8]. In a landmark clinical study focused on fibrosis reversion in CHB patients who received entecavir therapy, only $60 \%$ of biopsies were longer than $1.0 \mathrm{~cm}$ [9]. A systematic review reported the mean biopsy length was only $17.7 \pm 5.8 \mathrm{~mm}[10]$. Besides, many studies using liver biopsy as gold standard have not shown the data of biopsy length $[11,12]$.

An effective way to improve the diagnostic value in short liver biopsy samples is needed. In recent years, image morphometric analysis of liver biopsy sample has been applied to quantify the extent of liver fibrosis. qFibrosis (SHG/TPEF based), a structure-based quantitative assessment method, has been recently demonstrated to have a better performance for diagnosis of liver fibrosis compared with traditional collagen proportionate area (CPA) measurement [13]. Due to the comprehensive quantitation of collagen structure features and collagen spatial distribution, qFibrosis was shown to be less sensitive than CPA to sampling size in animal models.

Our aims in this study were: (1) to evaluate the diagnostic value of qFibrosis (SHG/TPEF) measurements for "short" biopsy samples, and (2) to illustrate why this technique is less sensitive to sample size compared to routine CPA measurements.

\section{Materials and methods}

\section{Liver biopsies}

Clinical biopsy samples were retrospectively extracted from a prospective HBV-related fibrosis/cirrhosis cohort study. The study cohort has been described previously [14]. In brief, the inclusions of recruitment were as follows: treatment-naive patients aged 18-65 years, positive for hepatitis B surface antigen (HBsAg) more than 6 months, HBV DNA levels higher than $20,000 \mathrm{IU} / \mathrm{mL}$ (positive for $\mathrm{HBeAg}$ ) or $2000 \mathrm{IU} / \mathrm{mL}$ (negative for $\mathrm{HBe} A g$ ), liver biopsy performed at baseline or week 78 after treatment.

Percutaneous needle biopsies were obtained with realtime ultrasound guidance. Tissue samples were fixed in formalin, embedded in paraffin, sectioned at $5 \mu \mathrm{m}$. All samples were stained with hematoxylin and eosin, Masson's trichrome and reticulin for standard histological assessment. One unstained section from each biopsy was evaluated by SHG/TPEF-based imaging. Biopsy length and fragmentation were documented.

Two senior pathologists (TLW and HL), who were blinded to all data, independently evaluated all the liver biopsy samples using METAVIR scoring system (F0, no fibrosis; F1, portal fibrosis without septa; F2, portal fibrosis with rare septa; F3, numerous septa without cirrhosis; F4, cirrhosis) [15]. Discordant cases will be reviewed again to achieve consensus.

\section{Image analysis}

Biopsies were imaged by second harmonic generation/twophoton excitation fluorescence (SHG/TPEF) microscopy [16]. A total of 101 collagen features were extracted from the SHG images and then the features were normalized by the tissue area. Fifteen collagen architectural features, previously identified as meaningful, were quantified and combined into a single qFibrosis score, as described in our previous study [16].

\section{Construction of virtual biopsy specimens}

All biopsies $\geq 1.5 \mathrm{~cm}$ in length were used to construct virtual biopsy specimens. Samples with $\leq 2$ fragments (one of the samples no shorter than $1.5 \mathrm{~cm}$ ) were used to construct "randomly defined" virtual biopsy specimens. Biopsies with more than two fragments were defined by the fractures ("fracture defined"). Detailed study design is shown in Fig. 1.

For randomly defined virtual biopsy, the first step was to define the starting point, and second step was to decide the specific length from 0.1 to $1.4 \mathrm{~cm}$, all based on a random number generator. Finally, all of virtual biopsies were randomly constructed into different lengths from 0.1 to $1.4 \mathrm{~cm}$ long using an image processing tool with precise calibration. Meanwhile, the fragmented biopsies were constructed into virtual biopsies of different lengths from 0.2 to $1.5 \mathrm{~cm}$ long. At final, all these samples were classified into four groups: $\leq 0.5-\mathrm{cm}, 0.5-1.0-\mathrm{cm}, 1.0-1.5-\mathrm{cm}$, and $\geq 1.5-\mathrm{cm}$ specimens.

The qFibrosis score was determined for each virtual biopsy specimen. The qFibrosis score of the entire tissue section was set as the reference of each case. For each virtual biopsy, the deviation of the qFibrosis score ( $\Delta$ qFibrosis) was defined as the absolute value of the difference between the score on a virtual biopsy and the score for the entire (reference) biopsy sample. A deviation within 0.50 was set as clinically acceptable error.

\section{Statistical analysis}

Numerical variables were expressed as median with the interquartile range and categorical data as number with frequencies. Intraclass correlation coefficient (ICC) was performed to assess the degree of consistency. $\Delta$ qFibrosis was defined as the absolute value of the deviation of $\mathrm{qFi}$ brosis scores between a virtual biopsy and the entire biopsy. Equivalence test was used to compare $\Delta$ qFibrosis with the clinically acceptable error (deviation of 0.50 ) in each group. The equivalence between the short biopsy samples and 


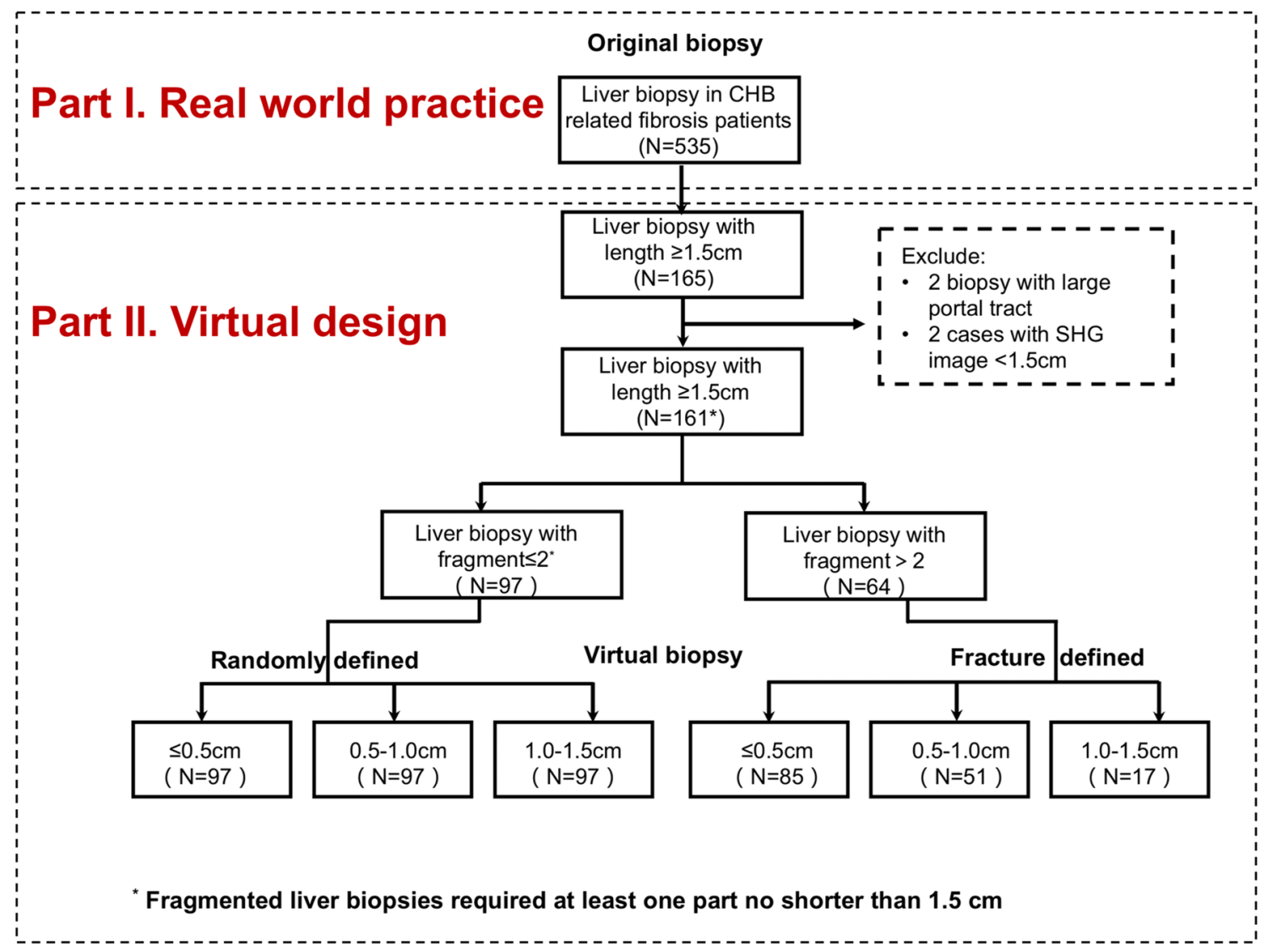

Fig. 1 Flow chart of study design. Part I: real-world practice; Part II: construction of virtual liver biopsies

" $\geq 1.5$-cm"-long specimens was confirmed if $\Delta \mathrm{qFibrosis}$ fell within 0.5 of the score. Continuous variables were compared using one-way ANOVA or Kruskal-Wallis test. Correlations were evaluated by Spearman's rank correlation. Equivalence test was performed with SAS 9.4; the other analyses were performed with SPSS 22.0. Two-sided $p$ values $<0.05$ were considered statistically significant.

\section{Results}

A total of 535 biopsy samples with evaluable fibrosis stage were retrospectively extracted from the prospective cohort study. The prevalence of fibrosis stages was $35.1 \%$ for $\mathrm{F} 1$ $(n=188), 30.1 \%$ for $\mathrm{F} 2(n=161), 19.1 \%$ for $\mathrm{F} 3(n=102)$ and $15.7 \%$ for $\mathrm{F} 4(n=84)$.

According to the length of liver biopsy, $30.8 \%$ of the samples were $\geq 1.5 \mathrm{~cm}$ long (165 cases, median length $1.7 \mathrm{~cm}$ ), $38.5 \%$ were $1.0-1.5 \mathrm{~cm}$ long (206 cases, median length $1.2 \mathrm{~cm}$ ), $26.2 \%$ were $0.5-1.0 \mathrm{~cm}$ long (140 cases, median length $0.9 \mathrm{~cm}$ ), and $4.5 \%$ were $\leq 0.5 \mathrm{~cm}$ long (24 cases, median length $0.5 \mathrm{~cm})$. A total of 165 (30.8\%) samples with biopsy length $\geq 1.5 \mathrm{~cm}$ were considered as qualified biopsy samples. The remaining biopsies $(69.2 \%)<1.5 \mathrm{~cm}$ were defined as unqualified or "short" biopsy samples. The distribution of fibrosis stage according to the biopsy length is shown in Table 1.

\section{Influence of biopsy length on the evaluation for fibrosis by qFibrosis for "short" samples in clinical practice: qFibrosis was good, especially in biopsy samples longer than $0.5 \mathrm{~cm}$}

First, we investigated the influence of biopsy length on the value of qFibrosis in differentiating fibrosis staging in clinical practice (Fig. 1, Part I). With fibrosis progression, qFibrosis score increased significantly both in $\geq 1.5-\mathrm{cm}$, 1.0-1.5-cm, and 0.5-1.0-cm specimens $(p<0.05)$, compared with $\leq 0.5-\mathrm{cm}$ specimens $(p>0.05)$ (Fig. 2a-d). As shown in Fig. $2 \mathrm{a}-\mathrm{d}$, the Spearman's correlation coefficient was $0.729,0.720,0.736$ in $0.5-1.0-\mathrm{cm}, 1.0-1.5-\mathrm{cm}$, and $\geq 1.5-\mathrm{cm}$ specimens, respectively, all $p$ values $<0.05$. qFibrosis had a significant correlation with fibrosis stage in biopsy samples longer than $0.5 \mathrm{~cm}$.

Interestingly, Fig. 2e shows the qFibrosis score might be higher in $\leq 0.5$-cm-long specimens than the other three 
Table 1 The distribution of biopsy length in biopsy specimens quantified by SHG/ TPEF technology-based microscope and evaluated by Metavir stage

\begin{tabular}{lcccrr}
\hline Biopsy length group & Total & \multicolumn{4}{l}{ Proportion of METAVIR fibrosis stage, $n(\%)$} \\
\cline { 3 - 6 } & & $F 1$ & $F 2$ & $F 3$ & \multicolumn{1}{l}{$F 4$} \\
\hline$\leq 0.5 \mathrm{~cm}, n(\%)$ & $24(4.5)$ & $8(1.5)$ & $6(1.1)$ & $7(1.3)$ & $3(0.6)$ \\
$0.5-1.0 \mathrm{~cm}, n(\%)$ & $140(26.2)$ & $62(11.6)$ & $35(6.5)$ & $23(4.3)$ & $20(3.7)$ \\
$1.0-1.5 \mathrm{~cm}, n(\%)$ & $206(38.5)$ & $58(10.8)$ & $67(12.5)$ & $38(7.1)$ & $43(8.0)$ \\
$\geq 1.5 \mathrm{~cm}, n(\%)$ & $165(30.8)$ & $60(11.2)$ & $53(9.9)$ & $34(6.4)$ & $18(3.4)$ \\
Total, $n(\%)$ & $535(100)$ & $188(35.1)$ & $161(30.1)$ & $102(19.1)$ & $84(15.7)$ \\
\hline
\end{tabular}

Data presented as number (percent)

b

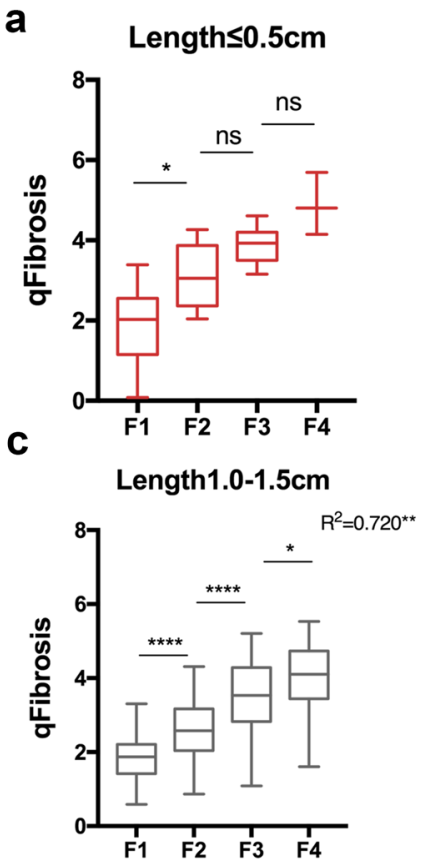

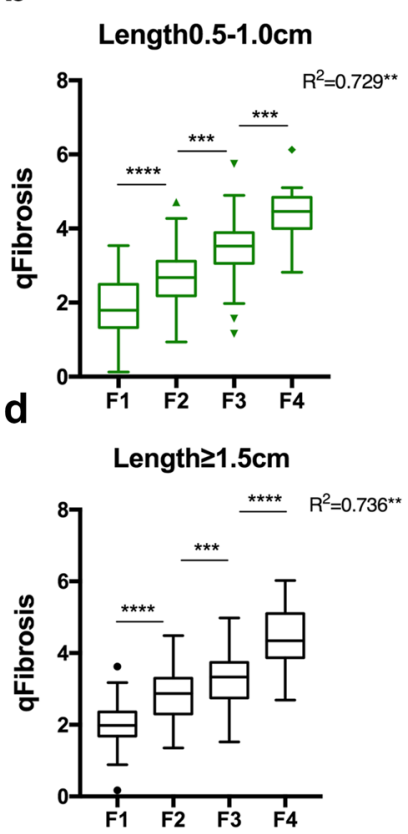

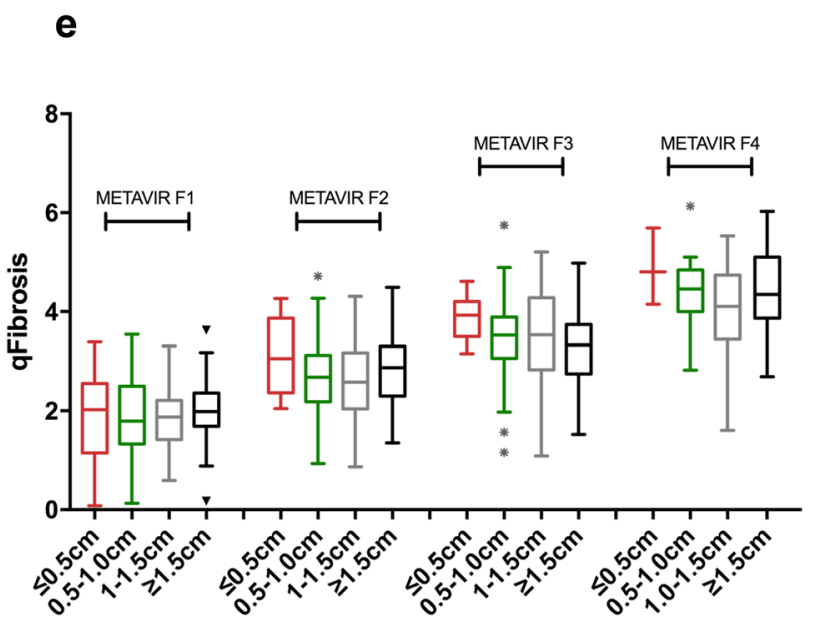

Fig. 2 Correlations of qFibrosis scores and METAVIR fibrosis score in original biopsies based on length. a Correlations of $\mathrm{qFi}$ brosis with METAVIR fibrosis stage in $\leq 0.5$-cm-long specimens $(p>0.05)$. b Correlations of qFibrosis with METAVIR fibrosis stage in $0.5-1.0-\mathrm{cm}-$ long specimens $(p<0.001, \gamma \mathrm{s}=0.729)$. $\mathbf{c}$ Correlations

groups in each fibrosis stage, though there was no significant difference. Figure 3 illustrates the images of representative biopsy samples with different sample size in each fibrosis stage. The qFibrosis score was slightly higher in $\leq 0.5-\mathrm{cm}$ specimens (Fig. 3a) than the other three groups at the same fibrosis stage (Fig. 3b-d).

\section{The influence of biopsy length on qFibrosis score by comparing "virtual" biopsy samples with original biopsy}

Then, to clarify the diagnostic value of qFibrosis score in differentiate fibrosis influenced by biopsy length, a total of 444 virtual biopsy samples were obtained from 161 qualified biopsies ( $\geq 1.5$-cm-long specimens, excluding two biopsies of qFibrosis with METAVIR fibrosis stage in 1.0-1.5-cm-long specimens $(p<0.001, \gamma s=0.720)$. d Correlations of qFibrosis with METAVIR fibrosis stage in $\geq 1.5$-cm-long specimens $(p<0.001, \gamma s=0.736$ ). e Distribution of $\mathrm{qFibrosis}$ with fibrosis progression according to biopsy length. $* * * * p<0.0001$

with large portal tract, two cases due to pathological process made the effective length of biopsy in SHG image $<1.5 \mathrm{~cm}$ ). The process of virtual liver biopsy construction is shown in Fig. 1, Part II.

qFibrosis score was less sensitive to biopsy length than CPA. For "randomly defined" virtual biopsy specimens, the relative deviation of qFibrosis against the original sample was gradually decreased from $\leq 0.5-\mathrm{cm}$-long specimens to $1.0-1.5-\mathrm{cm}$-long specimens and was smaller than that of CPA for each group (all $p$ values $<0.001$, Supplementary Fig. 1).

In "randomly defined" virtual biopsy samples, qFibrosis score for the assessment of liver fibrosis was equivalent between $0.5-, 1.0-\mathrm{cm}$ and $\geq 1.5-\mathrm{cm}$ specimens, as well as $1.0-1.5-\mathrm{cm}$ and $\geq 1.5-\mathrm{cm}$ specimens (all $p$ values $<0.001$ ), 


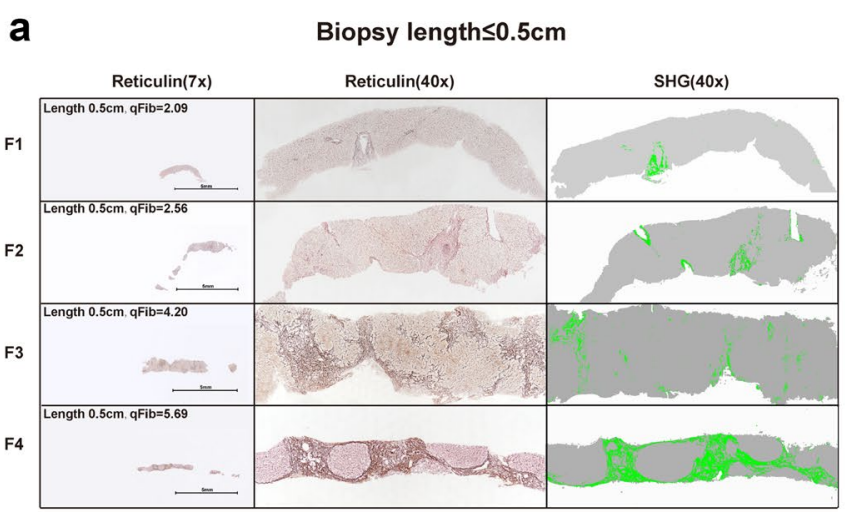

C

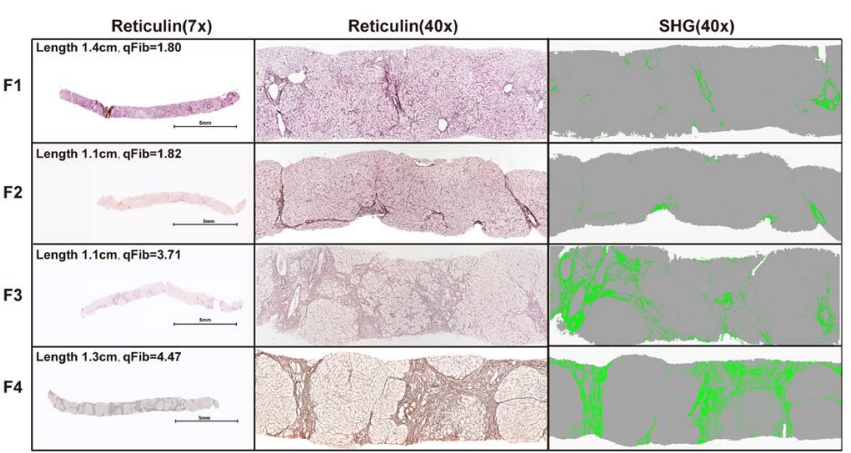

Fig. 3 Representative liver biopsy samples with various severity of fibrosis according to different biopsy length. The right panel showed the preview of liver biopsy (Reticulin, 7X). The middle panel indicated the typical histological feature (reticulin, 40X). The left panel showed the image analysis results of liver biopsy by SHG/ TPEF-based technology (pseudo-color, green stands for collagen fiber acquired by SHG signal, gray stands for parenchyma acquired by TPEF signal). a The reticulin staining and SHG/TPEF image in $\leq 0.5$-cm-long specimens. qFibrosis score of F1-F4 stage was

whereas not in $\leq 0.5-\mathrm{cm}$ specimens. The proportion of absolute value of $\Delta \mathrm{qFibrosis}$ within 0.50 was $55.7,90.7$ and $94.8 \%$ in $\leq 0.5-\mathrm{cm}, 0.5-1.0-\mathrm{cm}$ and $1.0-1.5-\mathrm{cm}$ specimens, respectively. The distribution of absolute deviation is shown in Fig. 4. In total, the mean absolute deviation was 0.53 in $\leq 0.5-\mathrm{cm}$ specimens, 0.27 in $0.5-1.0-\mathrm{cm}$ specimens, and 0.22 in $1.0-1.5-\mathrm{cm}$ specimens. The standard deviation decreased as biopsy size increased $(\mathrm{SD}=0.44,0.20,0.16$ in $\leq 0.5-\mathrm{cm}, 0.5-1.0-\mathrm{cm}$ and $1.0-1.5-\mathrm{cm}$ specimens, respectively). According to the subgroup analysis, the difference existed from F1 through F4 stages (Fig. 4b-e).

qFibrosis could distinguish the METAVIR stages well both in $0.5-1.0-\mathrm{cm}$ and $1.0-1.5-\mathrm{cm}$ specimens (AUC: $0.78-0.88$ ), as shown by ROC curve analysis (Supplementary Fig. 2).

For comparison, the distribution of absolute value of $\Delta \mathrm{qFibrosis}$ in virtual samples acquired from fractured liver biopsies is shown in Supplementary Fig. 3. qFibrosis score was equivalent between $0.5-, 1.0-\mathrm{cm}$ and $\geq 1.5-\mathrm{cm}$ specimens,
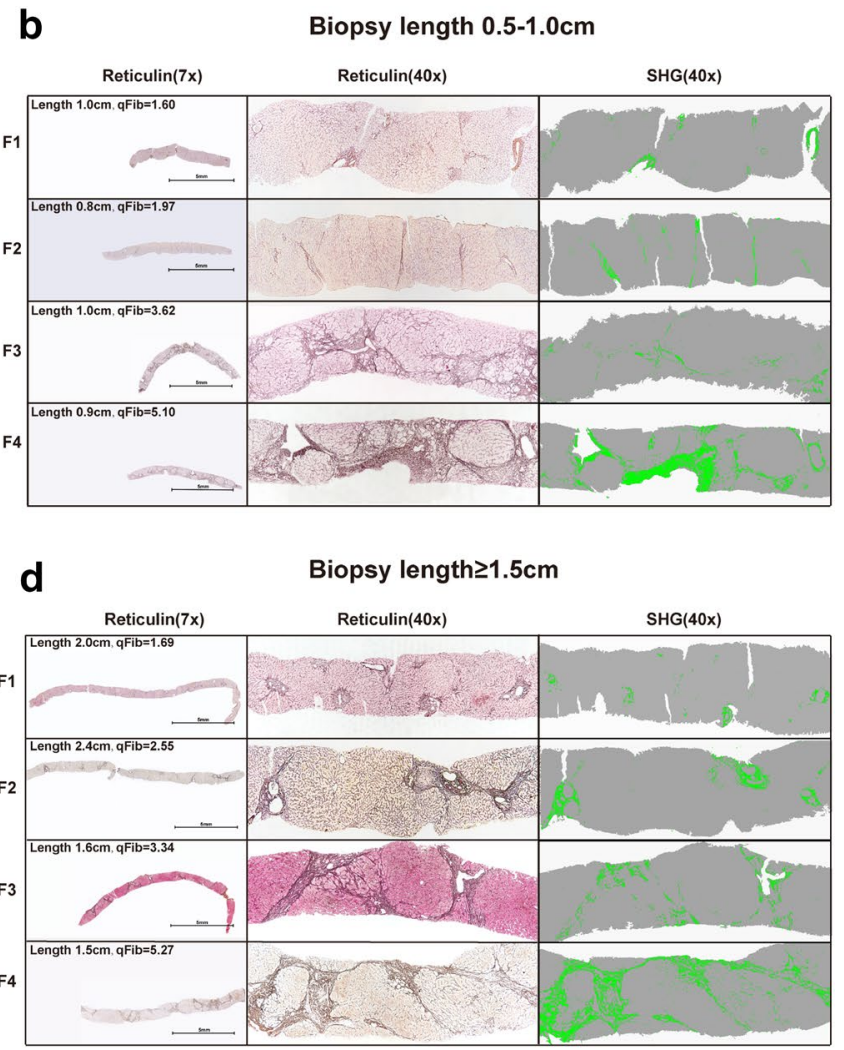

$2.09,2.56,4.20$ and 5.69 , respectively. b The reticulin staining and SHG/TPEF image in 0.5-1.0-cm-long specimens. qFibrosis score of F1-F4 stage was $1.60,1.97,3.62$ and 5.10, respectively. c The reticulin staining and SHG/TPEF image in $1.0-1.5-\mathrm{cm}-$ long specimens. qFibrosis score of F1-F4 stage was 1.80, 1.82, 3.71 and 4.47, respectively. d The reticulin staining and SHG/TPEF image in $\geq 1.5-\mathrm{cm}-$ long specimens. qFibrosis score of F1-F4 stage was 1.69, 2.55, 3.34 and 5.27 , respectively

as well as $1.0-1.5-\mathrm{cm}$ and $\geq 1.5-\mathrm{cm}$ specimens, whereas not in $\leq 0.5$-cm specimens. Examples of representative liver samples are shown in Fig. 5. As for mild fibrosis, the qFibrosis score was 2.60, and it remains at 2.44-2.73 in the longer fragments part of these samples, and reduced to 2.19 in samples shorter than $0.5 \mathrm{~cm}$. In advanced fibrosis, we can also see the same trend. The absolute value of $\Delta \mathrm{qFibrosis}$ was higher when sample shorter than $0.5 \mathrm{~cm}$.

Therefore, qFibrosis is relatively accurate in the evaluation of liver fibrosis in short samples if the length is greater than $0.5 \mathrm{~cm}$.

\section{The reason that qFibrosis overcomes the drawbacks of small sample size: additional parameters are considered compared to routine collagen proportionate area calculation}

To further explore why qFibrosis scores show good performance in evaluating fibrosis, we investigated the value 


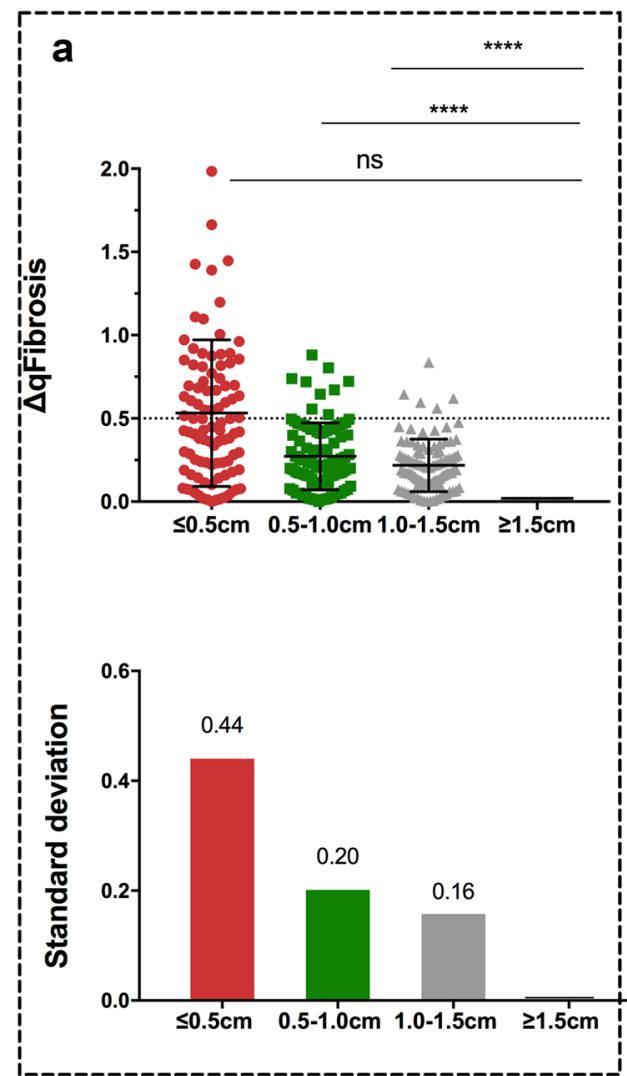

Fig. 4 Box plot showing the deviation (absolute value of $\Delta$ qFibrosis) in randomly defined virtual biopsies. $\Delta$ FFibrosis in $\geq 1.5-\mathrm{cm}$ group, shown as 0 , means a reference value for the other three groups. a The distribution of deviation in virtual biopsies with all spectrum of fibrosis stage according to biopsy length. $* * * * p<0.0001$. b The distribution of deviation in virtual biopsies with METAVIR stage F1 according to biopsy length. $* * * * p<0.0001$. c The distribution

of the additional parameters included in the calculation of the qFibrosis scores. Intraclass correlation coefficient of a possible 98 candidate parameters are shown in a heatmap (Fig. 6a). Among these candidate parameters, in samples of $0.5-1.0 \mathrm{~cm}$ and $1.0-1.5 \mathrm{~cm}$ length, 21 of these parameters had good consistency with results from larger specimens $(\geq 1.5 \mathrm{~cm})($ ICC $>0.90$, Supplementary Table 1$)$. Quantitative diagrams of many of the parameters are shown in Fig. 6b. In summary, the most consistent parameters were: the number of cross-linked collagen fibers, the total and aggregated collagen proportionate area, the number, length, width, and perimeter of collagen strings. It should be noted that the definition of cross-linked collagen fibers in the qFibrosis nomenclature bears no relation to collagen cross-linking at the molecular level used elsewhere in the hepatic fibrosis literature; it refers to the physical contacts of the fibers.

Considering the spatial distribution of the respective collagen patterns, portal area is susceptible to the change of biopsy length, followed by fibrillar area and septal area. of deviation in virtual biopsies with METAVIR stage F2 according to biopsy length. ****p $p<0.0001$. d The distribution of deviation in virtual biopsies with METAVIR stage F3 according to biopsy length. $* * * * p<0.0001$. e The distribution of deviation in virtual biopsies with METAVIR stage F4 according to biopsy length. $* * * p<0.001$. $* * * * p<0.0001 ; \mathrm{NS}, p>0.05$

AUROC of the consistent parameters in septal and fibrillar areas are shown in Supplementary Table 2. Compared with portal area, quantitative characters in the septal and fibrillar areas could be meaningful in diagnosing the severity of liver fibrosis. Detailed change of CPA in each area, the representative feature, is shown in Supplementary Fig. 4. The change percentage of CPA of total area, septal area and fibrillar area has no significant difference in $0.5-1.0-\mathrm{cm}$ and $1.0-1.5-\mathrm{cm}$ groups, whereas, not in portal area.

Among the 21 most consistent parameters, 9 are used to establish qfibrosis score, accounting for $60 \%$ of parameters of qFibrosis scores, perhaps illustrating why qFibrosis can minimize errors induced by small sample size.

\section{Discussion}

In this study, we demonstrated that biopsy samples longer than $0.5 \mathrm{~cm}$ are able to deliver a reliable quantitative assessment of fibrosis using the fully quantitative method 


\section{a Mild fibrosis}

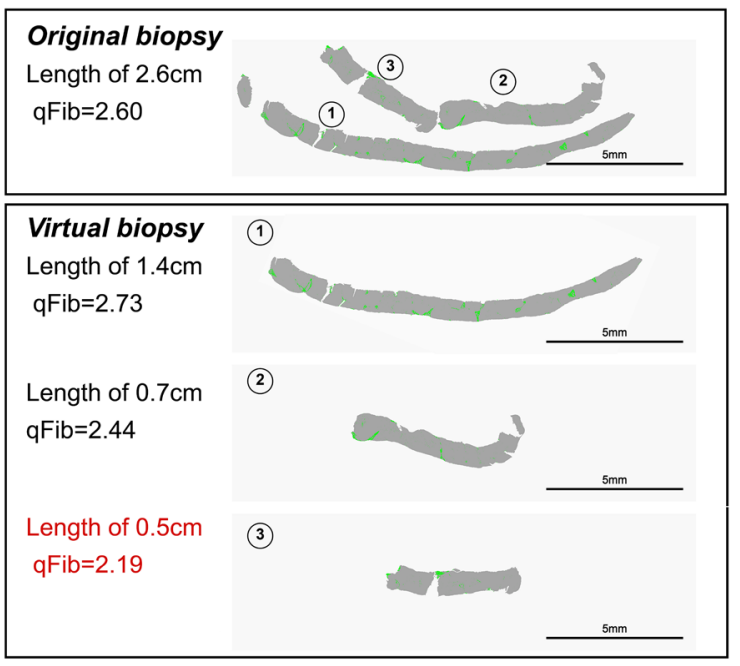

Fig. 5 Processed imaging of liver biopsies longer than $1.5 \mathrm{~cm}$ with fragmentation showing the deviation in $\leq 0.5$-cm-long specimen was higher than the other two groups. Virtual biopsies were constructed along the fractures (pseudo-color, green stands for collagen fiber acquired by SHG signal, gray stands for parenchyma acquired by TPEF signal). a Representative biopsy samples with mild fibrosis (F1). qFibrosis score of the original biopsy is 2.60. After virtual image selection, in biopsy longer than $0.5 \mathrm{~cm}$, the qFibrosis score is

of qFibrosis. The histological features in septal and fibrillar area were insusceptible to sample size, illustrating the reason why qFibrosis has an advantage in resolving the issue of sampling error.

The impact of biopsy length on quantitative fibrosis score (qFibrosis) in real-world practice of CHB patients was first analyzed. qFibrosis showed a very good performance in reflecting the severity of fibrosis in "short" biopsy samples if it is longer than $0.5 \mathrm{~cm}$. It is not clear whether this good performance is caused by underestimation of METAVIR fibrosis stage or improved detection of fibrosis with the qFibrosis technology. To clarify this issue, we constructed "virtual biopsy samples" from actual biopsies $\geq 1.5 \mathrm{~cm}$ in length. These "virtual" biopsies simulated the so-called "short" biopsy specimens. The deviation and consistency of each biopsy were individually calculated by comparing with the biopsies longer than $1.5 \mathrm{~cm}$. According to our analysis, the absolute value of $\Delta q$ Fibrosis maintained a low level in liver biopsies if they were at least $0.5 \mathrm{~cm}$ in length. Similar to our results, another study has shown recently that qFibrosis was less sensitive to sample size than CPA [13].

The reason why this SHG/TPEF-based image analysis could reliably reflect the severity of liver fibrosis in small biopsies is the consistency of the established quantitative parameters between long and short biopsy samples. In our study, we found the stable features included: the number of

\section{b Advanced fibrosis}

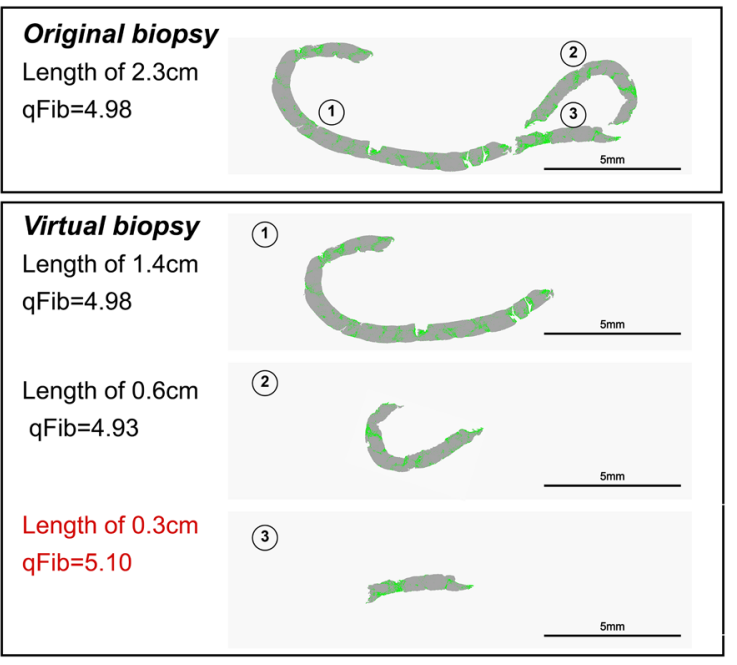

maintained at 2.73 and 2.44, respectively. In the smallest fragmented biopsy samples shorter than $0.5 \mathrm{~cm}$, the qFibrosis score decreased to 2.19. Scale bar $=5 \mathrm{~mm}$. b Representative biopsy samples with advanced fibrosis (F3). qFibrosis score of the original biopsy is 4.98. After image selection, in biopsy longer than $0.5 \mathrm{~cm}$, the qFibrosis score is maintained at 4.98 and 4.93 , respectively. In the smallest fragmented biopsy samples shorter than $0.5 \mathrm{~cm}$, the qFibrosis score increased to 5.10 . Scale bar $=5 \mathrm{~mm}$

cross-linked collagen fibers, the total and aggregated collagen proportionate area, the length, width, area and perimeter of collagen strings in total. qFibrosis, SHG/TPEF-based image technology, incorporates the multiple spatial architectural collagen features, giving the reason why qFibrosis can minimize errors induced by small sample size.

Interestingly, when considering the spatial characteristics of collagen pattern, features in septal and fibrillar areas were less sensitive to a reduced biopsy length, compared to portal features. Quantitative characters in the septal and fibrillar areas are meaningful in diagnosing the severity of liver fibrosis compared with the features in portal area. This sensitivity in small biopsies may be explained by reduced portal tract number while histological features in septal and fibrillar areas remain relatively constant because of their distributed location.

These favorable results with qFibrosis are in contrast to those from studies with traditional methods demonstrating substantial sampling error $[4,6,7,17,18]$. For these methods, a relatively generous 2 -cm-long sample is now recommended $[8,19]$, leading to increased peri-operative risk [10, 20]. Traditional methods are also affected by lack of expertise and poor interobserver agreement, while qFibrosis is totally independent of these effects [21].

Traditional staging methods have been compared to standard digital image analysis, focusing on the issue of sampling error. [22] In a study of cirrhotic transplant tissue, 


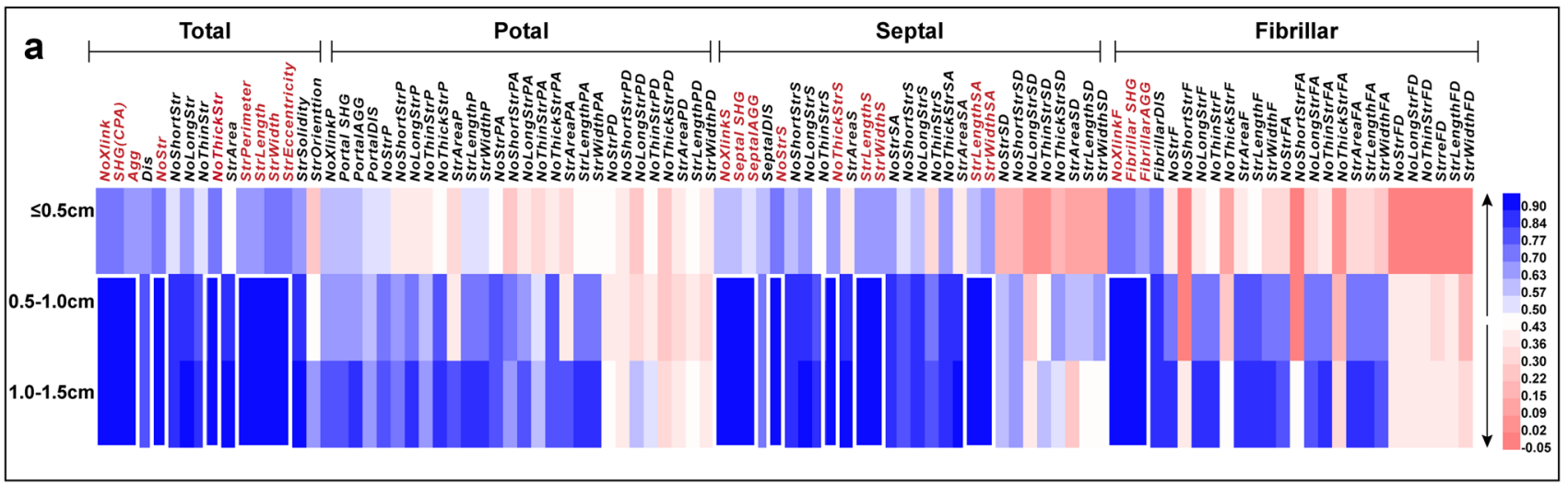

b

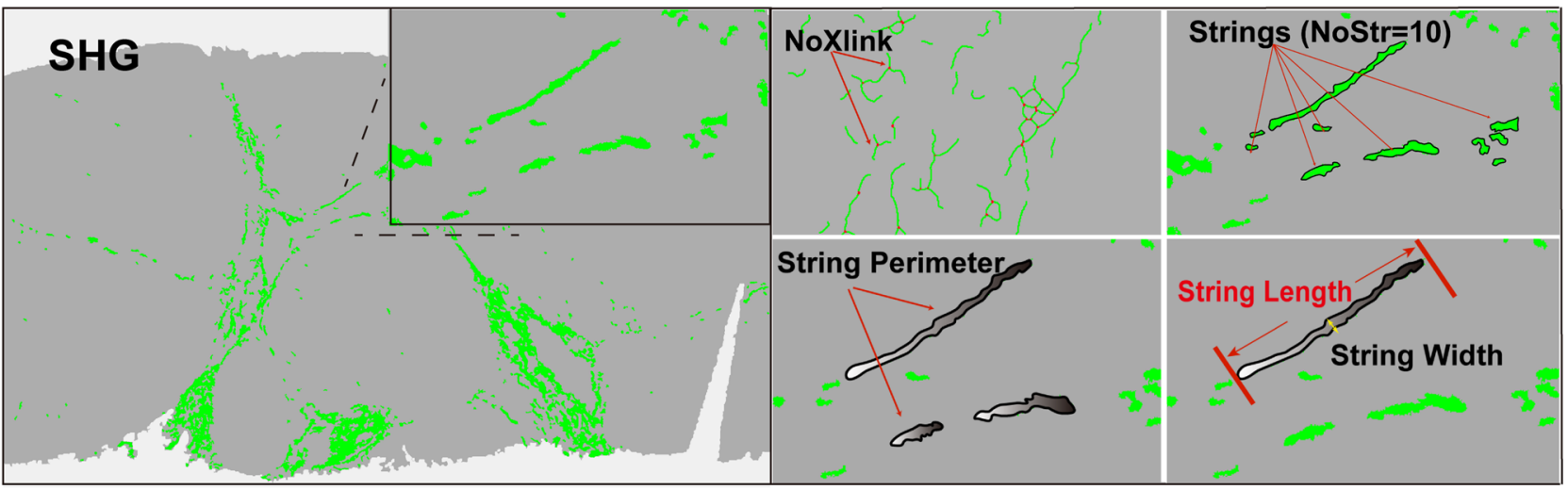

Fig. 6 Heatmap and quantitative diagram of morphological parameters by SHG/TPEF technology in virtual biopsies. a Heatmap of the intraclass correlation efficient between "unqualified" biopsy samples and "qualified" biopsy samples of each quantitative parameters; b Quantitative diagrams of many of the morphological features which were less susceptible to biopsy length, including collagen proportion area by, length, width and contour perimeter of collagen string,

Hall et al. concluded that, to achieve the $75 \%$ probability that CPA of a virtual biopsy will be within 5\% of the reference CPA, a length of 15-20 mm was required [22]. This requirement does not show an advantage of CPA over traditional histological analysis in the issue of sampling error. Our results suggest that qFibrosis methodology is more resistant to sampling error compared to CPA measurements by standard digital image analysis methods and the benefits are extended across the fibrosis range of F1-F4.

The clinical implications of these results might be profound, as shown in Supplementary Fig. 5. In the past, the guidelines have recommended liver biopsy length be at least $1.5 \mathrm{~cm}$, to avoid sampling error, while our data with qFibrosis suggest that $0.5 \mathrm{~cm}$ may be sufficient for this purpose.

The strengths of our study are as follows: (1) the quantitative method we used is more sensitive and specific than traditional digital image analysis. It has been demonstrated to have better diagnostic value for discriminating adjacent stages even in early fibrosis stage compared with traditional cross-linked collagen strings. SHG, total collagen proportionate area quantified by SHG/TPEF; Agg, aggregated collagen; Dis, distributed collagen; NoXlink, number of cross-linked collagen strings; NoStr, number of collagen strings; StrLength, the length of collagen strings; StrWidth, the width of collagen strings; StrEccentricity, the eccentricity of collagen strings; StrSolidity, the solidity of collagen strings; StrPerimeter, the perimeter of collagen strings

digital image analysis. (2) The samples used in our study were liver biopsies, as opposed to samples obtained from liver transplantation with a limited range of stages. The simulation of "short" biopsy sample was close to the actual possibility. (3) Our cohort, including 165 biopsies, was larger than the other studies focused on the same issue.

There are still some limitations of our study. First, liver biopsy sample of cirrhosis is more easily to be fragmented, which is a major obstacle in the diagnosis of cirrhosis. However, the proportion of cirrhotic specimens is relatively small in our study, so the diagnostic value of qFibrosis in short cirrhotic samples needs to be further verified in larger number of samples. Second, all the liver biopsies were obtained from CHB patients in our study. The histological pattern of fibrosis from other etiologies may be different from $\mathrm{CHB}$ patients, indicating the application of qFibrosis in "short" biopsies other than CHB needs to be further explored.

Despite these limitations, our study still demonstrated that SHG/TPEF-based technology, coupled with additional 
parameters discovered by machine learning (qFibrosis scores) showed good performance in the evaluation of CHBrelated liver fibrosis in short samples down to a lower limit of $0.5 \mathrm{~cm}$ in length. Future studies would be of great interest to search for further improvements in the diagnostic value of qFibrosis using small "unqualified" liver biopsies in the assessment of liver fibrosis.

Acknowledgements This study was funded by National Science and Technology Major Project (2018ZX10302-204), Key Project from Beijing Municipal Science and Technology Commission (D161100002716003), and National Natural Science Foundation of China (81670539).

Author contributions Study design: HY, XJO and JDJ. Data collection: YMS, JLZ, YWS, XNW, BQW, SYC, XJO. Liver biopsy assessment: TLW, HL. Statistical analysis: BQW, SSW. Manuscript writing: BQW. Critical revision of the manuscript: HY.

\section{Compliance with ethical standards}

Conflict of interest Bingqiong Wang, Yameng Sun, Jialing Zhou, Xiaoning Wu, Shuyan Chen, Yiwen Shi, Shanshan Wu, Hui Liu, Yayun Ren, Xiaojuan Ou, Jidong Jia, and Hong You declare that they have no conflict of interest.

Open Access This article is distributed under the terms of the Creative Commons Attribution 4.0 International License (http://creativeco mmons.org/licenses/by/4.0/), which permits unrestricted use, distribution, and reproduction in any medium, provided you give appropriate credit to the original author(s) and the source, provide a link to the Creative Commons license, and indicate if changes were made.

\section{References}

1. Hølund B, Poulsen H, Schlichting P. Reproducibility of liver biopsy diagnosis in relation to the size of the specimen. Scand $\mathbf{J}$ Gastroent. 1980;15:329-335

2. Schlichting P, Holund B, Poulsen H. Liver biopsy in chronic aggressive hepatitis. Diagnostic reproducibility in relation to size of specimen. Scand J Gastroenterol. 1983;18:27-32

3. Regev A, Berho M, Jeffers LJ, Milikowski C, Molina EG, Pyrsopoulos NT, Feng ZZ, et al. Sampling error and intraobserver variation in liver biopsy in patients with chronic HCV infection. Am J Gastroenterol. 2002;97:2614-2618

4. Colloredo G, Guido M, Sonzogni A, Leandro G. Impact of liver biopsy size on histological evaluation of chronic viral hepatitis: the smaller the sample, the milder the disease. J Hepatol. 2003;39:239-244

5. Bravo AA, Sheth SG, Chopra S. Liver biopsy. N Engl J Med. 2001;344:495-500

6. Schiano TD, Azeem S, Bodian CA, Bodenheimer HC Jr, Merati S, Thung SN, Hytiroglou P. Importance of specimen size in accurate needle liver biopsy evaluation of patients with chronic hepatitis C. Clin Gastroenterol Hepatol. 2005;3:930-935
7. Bedossa P, Dargere D, Paradis V. Sampling variability of liver fibrosis in chronic hepatitis C. Hepatology. 2003;38:1449-1457

8. Standish RA, Cholongitas E, Dhillon A, Burroughs AK, Dhillon AP. An appraisal of the histopathological assessment of liver fibrosis. Gut. 2006;55:569-578

9. Chang TT, Liaw YF, Wu SS, Schiff E, Han KH, Lai CL, et al. Long-term entecavir therapy results in the reversal of fibrosis/ cirrhosis and continued histological improvement in patients with chronic hepatitis B. Hepatology. 2010;52:886-893

10. Cholongitas E, Senzolo M, Standish R, Marelli L, Quaglia A, Patch D, et al. A systematic review of the quality of liver biopsy specimens. Am J Clin Pathol. 2006;125:710-721

11. Marcellin P, Gane E, Buti M, Afdhal N, Sievert W, Jacobson IM, et al. Regression of cirrhosis during treatment with tenofovir disoproxil fumarate for chronic hepatitis B: a 5-year open-label follow-up study. Lancet. 2013;381:468-475

12. Dienstag JL, Goldin RD, Heathcote EJ, Hann HW, Woessner M, Stephenson SL, et al. Histological outcome during long-term lamivudine therapy. Gastroenterology. 2003;124:105-117

13. Xu S, Wang Y, Tai DC, Wang S, Cheng CL, Peng Q, et al. qFibrosis: a fully-quantitative innovative method incorporating histological features to facilitate accurate fibrosis scoring in animal model and chronic hepatitis B patients. J Hepatol. 2014;61:260-269

14. Sun Y, Zhou J, Wang L, Wu X, Chen Y, Piao H, et al. New classification of liver biopsy assessment for fibrosis in chronic hepatitis B patients before and after treatment. Hepatology. 2017;65:1438-1450

15. Bedossa P, Poynard T. An algorithm for the grading of activity in chronic hepatitis C. The METAVIR Cooperative Study Group. Hepatology. 1996;24:289-293

16. Sun Y, Zhou J, Wu X, Chen Y, Piao H, Lu L, et al. Quantitative assessment of liver fibrosis (qFibrosis) reveals precise outcomes in Ishak "stable" patients on anti-HBV therapy. Sci Rep. 2018;8:2989

17. Scheuer PJ. Liver biopsy size matters in chronic hepatitis: bigger is better. Hepatology. 2003;38:1356-1358

18. Coral GP, Antunes AD, Serafini AP, Araujo FB, Mattos AA. Liver biopsy: importance of specimen size in the diagnosis and staging of chronic viral hepatitis. Rev Inst Med Trop Sao Paulo. 2016;58:10

19. Guido M, Rugge M. Liver biopsy sampling in chronic viral hepatitis. Semin Liver Dis. 2004;24:89-97

20. Guido M, Colloredo G, Fassan M, Rugge M. Clinical practice and ideal liver biopsy sampling standards: not just a matter of centimeters. J Hepatol. 2006;44:823-824

21. Rousselet MC, Michalak S, Dupre F, Croue A, Bedossa P, SaintAndre JP, et al. Sources of variability in histological scoring of chronic viral hepatitis. Hepatology. 2005;41:257-264

22. Hall AR, Tsochatzis E, Morris R, Burroughs AK, Dhillon AP. Sample size requirement for digital image analysis of collagen proportionate area in cirrhotic livers. Histopathology. 2013;62:421-430

Publisher's Note Springer Nature remains neutral with regard to jurisdictional claims in published maps and institutional affiliations. 